Case report

\title{
Isolated right adrenal metastasis from an invasive ductal breast carcinoma.
}

Taha Mohammed Hassan 1*, Al Emadi Mohammed Abdullah 1, Lotfy Mohammed 1, Mustafa Mohamed Naser 2, Omar Amany Mohammed Rabie 3, El Mahdi Hoda Saleh 3.

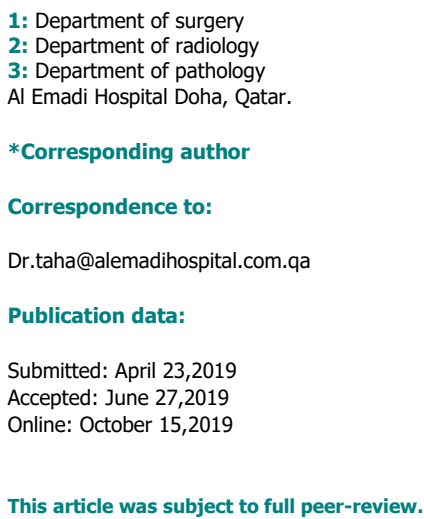

This art This is an open access article distributed under the terms of the Creative Commons Attribution Non4.0 (CCBY-NC) allowing to share and adapt.

Share: copy and redistribute the material in any medium or format.

Adapt:

the work provided must be properly cited and cannot be used for commercial purpose.

\section{Introduction}

Invasive ductal carcinoma (IDC) is the most common histopathological type of breast cancer, accounting for up to $85 \%$ of all invasive breast carcinomas [1]. It spreads usually to the bone first. Solitary metastasis is commonly located in the lung, liver or brain [2]. Adrenal glands locations are extremely rare [3]. We report a case of isolated metachronous right adrenal metastasis, diagnosed four years after breast IDC management. The aim is to highlight clinical, diagnostic and therapeutic characteristics of this entity.

\section{Case presentation}

We report a case of 53 years old female referred to surgery for right adrenal mass. On her history we noted a previous tumorectomy followed by chemo and radiotherapy for right breast invasive ductal carcinoma moderately differentiated Not Otherwise Specified (IDC-NOS). The TNM staging of the tumor was T2 NX MO. On the four-year follow-up consultation, an incidental right suprarenal tumor was detected by abdominal ultrasound. CT and MRI scan (Figure 1), confirmed the presence of a $5 \times 7 \mathrm{~cm}$ right adrenal gland mass. Endocrinological workup revealed that the tumor was not secreting. The patient underwent Laparoscopic Adrenalectomy which had to be converted to open, from which the patient made uneventful recovery. Gross examination revealed firm capsulated right adrenal mass, measuring $9 \times 7 \times 3 \mathrm{~cm}$ and about 123.5 grams in weight with cut sections showed heterogenous cystic appearance. Microscopic examination revealed infiltration by sheets, clusters and cords of malignant cells (figure 2a) with enlarged nuclei, prominent nucleoli and frequent mitosis. The tumor reached the surrounding fat with focal vascular invasion (figure 2b).Immunohistochemical staining revealed that tumor cells expressed diffuse positive staining with ER $(2+$ in $80 \%$ of tumor cells) (figure $4 a)$, PR ( $2+$ in $2 \%$ of tumor cells), GATA3 (figure 4b), $\mathrm{E}$-cadherin, $\mathrm{BCL}-2$, HER-2 is equivocal (2+) and Ki67 proliferation index was $30 \%$. Histopathological confrontation of both adrenal and breast specimens revealed identical morphological and immunohistochemical features and was consistent with metastatic mammary carcinoma. Thereafter, the patient received 6 cycles of chemotherapy followed by hormonal therapy and kept a regular follow up until now.

Citation: Taha MH, Al Emadi MA, Lotfy M, Mustafa MN, Omar A, El Mahdi H. Isolated right adrenal metastasis from an invasive ductal breast carcinoma. Junior medical research.2019;2(2): 10-13. Taha et al (c) All rights are reserved. 


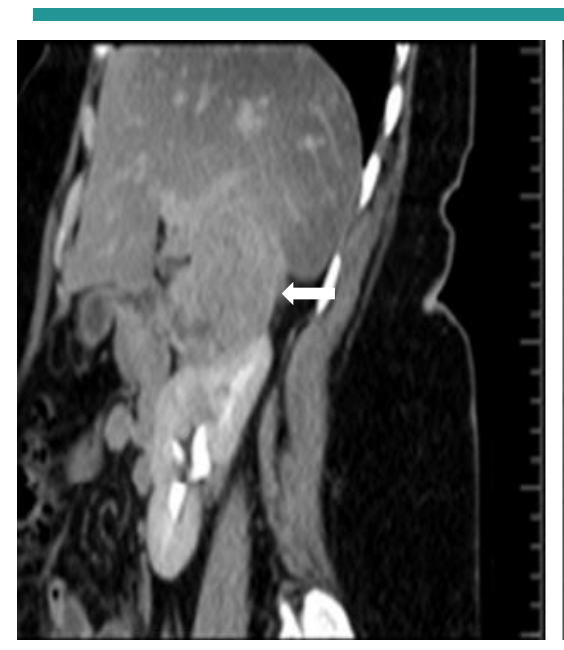

a

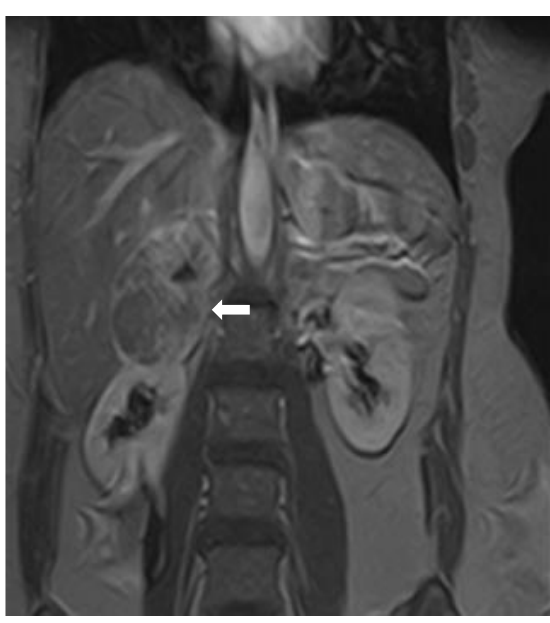

b

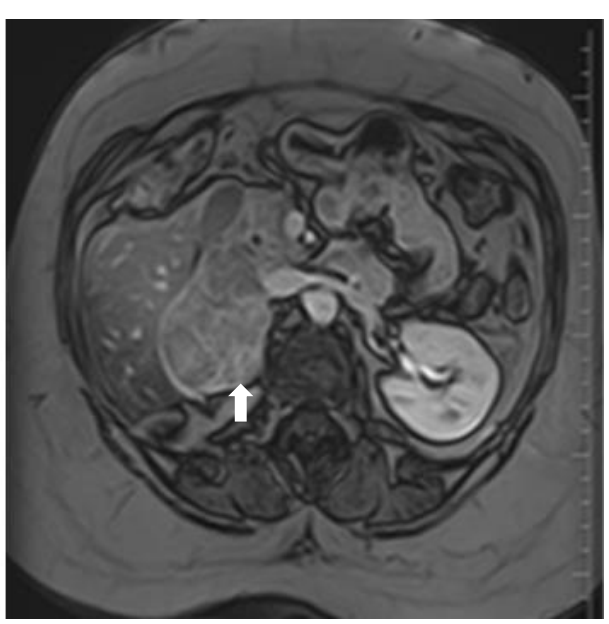

C

Figure1: Imaging features of the right incidentaloma.

(a) CT scan showed $5 \times 7 \times 4$ heterogenous mass of the right suprarenal region. $(b, c)$ right adrenal origin confirmed by MRI out phases showed a well-defined tumor arising from the adrenal gland with mass effect behavior on the right kidney
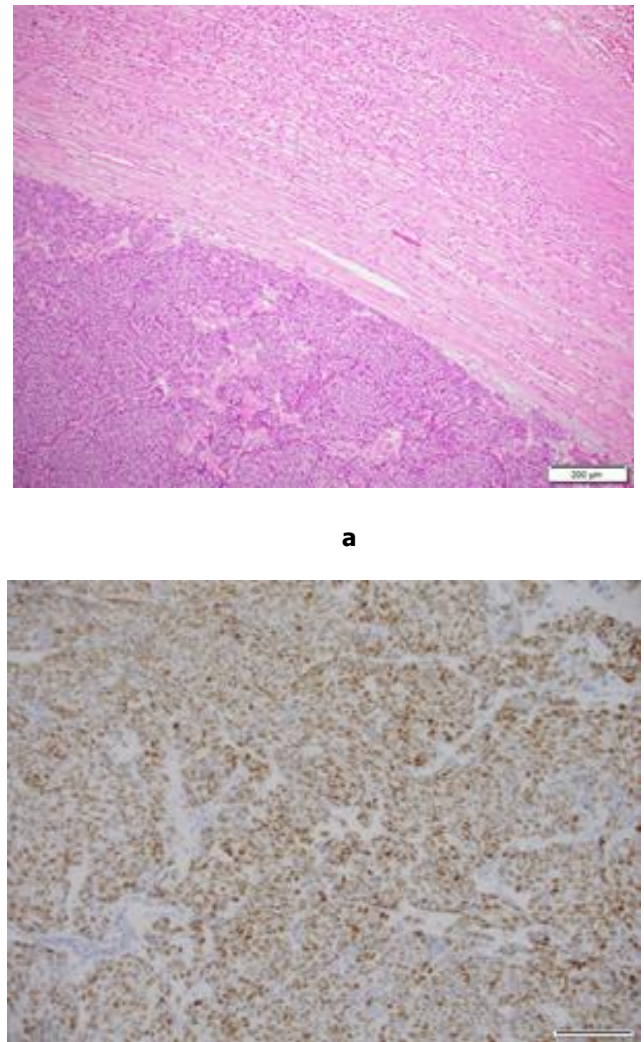

C

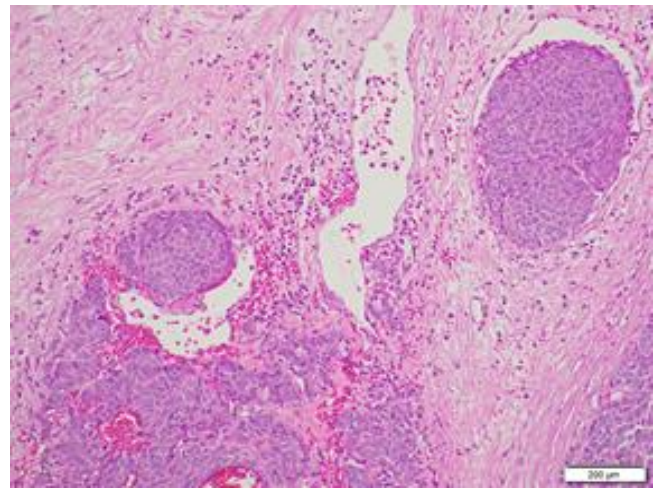

b

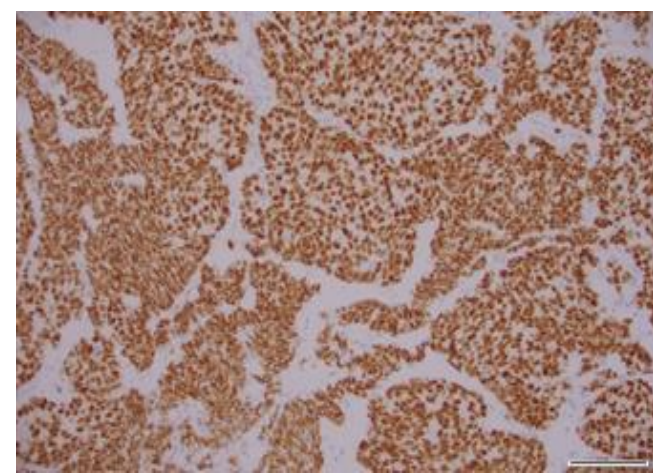

d

Figure 2: pathological examination

(a)Histology section shows adrenal tissue infiltrated by sheets of malignant cells ( $H$ and $E, x 200)$. (b) Histology section shows vascular invasion ( $H$ and $E$, x200). (c) Diffuse positive nuclear staining for ER (ER immunostaining, x200). (d) Diffuse positive nuclear staining for GATA3 (GATA3 immunostaining, x400).

Citation: Taha MH, Al Emadi MA, Lotfy M, Mustafa MN, Omar A, El Mahdi H. Isolated right adrenal metastasis from an invasive ductal breast carcinoma. Junior medical research.2019;2(2): 10-13. Taha et al (C) All rights are reserved. 


\section{Discussion}

The first published case of breast metastasis at adrenal gland was by Liu et al in 2010 [1]. Only eight cases of adrenal metastases from invasive breast carcinoma not otherwise specified were published up to date [2]. In six cases the metastasis was to the left adrenal gland $[1,2]$ and in only two cases metastasis was to the right adrenal gland [3] as our case. We have reported an incidental right adrenal metastasis which originated from moderately differentiated invasive ductal carcinoma managed four year before. Adrenal metastatic tumors are often misdiagnosed as primary adrenal tumors. CT scan and MRI are suitable methods for distinguishing between a metastatic and primary adrenal tumor [4,5]. Additionally, immunoassaying of tissue sections can confirm the primary site [6]. In our case immunoassaying confirmed the breast origin. Patients with adrenal metastasis usually are asymptomatic and start to express suprarenal gland disfunction only when most of the adrenal gland is replaced or destroyed $[7,8]$. Our patient underwent Laparoscopic Right Adrenalectomy which was converted to open due to the large size of the tumor. In eight published cases, one case was treated by Lapatinib ${ }^{\circledR}$ [9] and the remaining cases was treated by open or laparoscopic Adrenalectomy [9]. The ideal treatment options of this condition are still unclear because of the rarity of solitary adrenal metastases from mammary carcinoma. Metastatic breast cancer is often fatal and the treatment in most of the cases is mainly limited to palliation chemotherapy or hormonal treatment. However, laparoscopic adrenalectomy for solitary adrenal metastasis is considered convenient, and could lead to a longer survival in some patients $[10,11]$. Even though it is important to avoid port-site metastasis and carcinomatosis which may happen after the procedure. Laparoscopic adrenalectomy is widely applied to adrenal gland tumors. However, the gold standardization in adrenal cancer metastasis remains controversial [12].

\section{Conclusions}

Although adrenal metastasis of an IDC is very rare, the present case is the ninth reported breast cancer metastatic to the adrenal gland. Definite diagnosis can be established by CT, MRI and histopathological examination. Early recognition and complete adrenalectomy may lead to survival benefit.

\section{Conflict of interest: none}

\section{References}

[1]Liu XJ1, Shen P, Wang XF, Sun K, Sun FF. Solitary adrenal metastasis from invasive ductal breast cancer: an uncommon finding. World ] Surg Oncol. 2010; 8:7.

[2]Akhtar K, Sherwani R, Kahkhashan E. Carcinoma breast metastasis to the suprarenal gland: an unusual presentation. Pol J Pathol. 2012 ; 63: 284-5.

[3]Barros N.A, Ferreira, A., Rocha, M.J., et al. Unusual Breast Cancer Metastasis. BM] Case Reports. 2015; 43-45.

[4]Toikkanen S, Pylkkänen L, Joensuu H. Invasive lobular carcinoma of the breast has better short- and long-term survival than invasive ductal carcinoma. Br J Cancer. 1997; 76:1234-40.

[5]Fassnacht M, Kenn W, Allolio B. Adrenal tumors. How to establish malignancy. J Endocrinol Invest. 2004; 27:387-99.

[6]Takeda Y, Tsuta K, Shibuki Y. Analysis of expression patterns of breast cancer-specific markers (mammaglobin and gross cystic disease fluid protein 15) in lung and pleural tumors. Arch Pathol Lab Med. 2008; 132:239-43.

[7] He T, Liu J, Li Y, Jin LU, Sun S, Ni L, et al. Left adrenal gland metastasis of breast invasive ductal carcinoma: A case report. Mol Clin Oncol. 2016; 4:859-62.

[8]Suzuki H. Laparoscopic adrenalectomy for adrenal gland carcinoma and metastases. Curr Opin Urol. 2006; 16:47-53.

[9]Shamoun S, Shamout A. A Case Report of Two Primary Cancers, Breast Cancer with Adrenal Gland Metastatic and Second Primary Neuroendocrine Tumor in Colon, a Rare Case in Al-Bashir Hospital. Case Reports in Clinical Medicine. 2018; 7: 306-13.

[10]Tanvetyanon T, Robinson LA, Schell MJ. Outcomes of adrenalectomy for isolated synchronous versus metachronous adrenal metastases in non-small cell lung cancer: a systematic review and pooled analysis. J Clin Oncol. 2008; 26:1142-47.

[11]Zografos GN, Perysinakis I, Kyrodimou E, Kassi E, Kaltsas G. Surgical treatment of potentially primary malignant adrenal gland tumors: An unresolved issue. Hormones. 2015; 14:47-58.

[12]Shoji S, Usui Y, Nakano M, Hanai K, Sato H, Uchida T, et al. Surgical management of metastatic adrenal gland tumors: Decision-making factors in imaging. Oncol Lett. 2010; 1:967-71. 